\title{
Article \\ Lipid Dynamics in Diisobutylene-Maleic Acid (DIBMA) Lipid Particles in Presence of Sensory Rhodopsin II
}

\author{
Natalia Voskoboynikova ${ }^{1}\left(\right.$ ) , Philipp Orekhov ${ }^{2,3,4}{ }^{\circledR}$, Marine Bozdaganyan ${ }^{4,5}$, Felix Kodde ${ }^{1}$, Malte Rademacher ${ }^{1}$, \\ Maurice Schowe ${ }^{1}$, Annette Budke-Gieseking ${ }^{6}$, Britta Brickwedde ${ }^{6}$, Olympia-Ekaterini Psathaki ${ }^{6}$, \\ Armen Y. Mulkidjanian ${ }^{1,7}$, Katia Cosentino ${ }^{6}$, Konstantin V. Shaitan ${ }^{4,5} \mathbb{D}_{\mathbb{D}}$ and Heinz-Jürgen Steinhoff $1, * \mathbb{C}$
}

Citation: Voskoboynikova, N.; Orekhov, P.; Bozdaganyan, M.; Kodde, F.; Rademacher, $\mathrm{M}$. Schowe, M.; Budke-Gieseking, A.; Brickwedde, B.; Psathaki, O.-E.; Mulkidjanian, A.Y.; et al. Lipid Dynamics in Diisobutylene-Maleic Acid (DIBMA) Lipid Particles in Presence of Sensory Rhodopsin II. Int. J. Mol. Sci. 2021, 22, 2548. https://doi.org/ $10.3390 /$ ijms 22052548

Academic Editor: Laurence Lins

Received: 12 February 2021

Accepted: 26 February 2021

Published: 4 March 2021

Publisher's Note: MDPI stays neutral with regard to jurisdictional claims in published maps and institutional affiliations.

Copyright: (c) 2021 by the authors. Licensee MDPI, Basel, Switzerland. This article is an open access article distributed under the terms and conditions of the Creative Commons Attribution (CC BY) license (https:/ / creativecommons.org/licenses/by/ $4.0 /)$.
1 Department of Physics, University of Osnabrück, Barbarastrasse 7, D-49076 Osnabrück, Germany; nvoskobo@uni-osnabrueck.de (N.V.); fkodde@outlook.de (F.K.); mrademacher@uni-osnabrueck.de (M.R.); mschowe@uni-osnabrueck.de (M.S.); amulkid@uni-osnabrueck.de (A.Y.M.)

2 Moscow Institute of Physics and Technology, 141701 Dolgoprudny, Russia; ps.orekhov@gmail.com

3 Institute of Personalized Medicine, Sechenov University, 119146 Moscow, Russia

4 Department of Biology, Lomonosov Moscow State University, 119991 Moscow, Russia; m.bozdaganyan@gmail.com (M.B.); shaytan49@yandex.ru (K.V.S.)

5 N.N. Semenov Federal Research Center for Chemical Physics, Russian Academy of Sciences, 119991 Moscow, Russia

6 Department of Biology and Center for Cellular Nanoanalytics (CellNanOs), University of Osnabrück, Barbarastrasse 11, D-49076 Osnabrück, Germany; annbudke@uni-osnabrueck.de (A.B.-G.); bbrickwedde@uni-osnabrueck.de (B.B.); katherina.psathaki@uni-osnabrueck.de (O.-E.P.); kacosentino@uni-osnabrueck.de (K.C.)

7 Faculty of Bioengineering and Bioinformatics and Belozersky Institute of Physico-Chemical Biology, M.V. Lomonosov Moscow State University, 119234 Moscow, Russia

* Correspondence: hsteinho@uni-osnabrueck.de; Tel.: +49-541-969-2675

\begin{abstract}
Amphiphilic diisobutylene/maleic acid (DIBMA) copolymers extract lipid-encased membrane proteins from lipid bilayers in a detergent-free manner, yielding nanosized, discoidal DIBMA lipid particles (DIBMALPs). Depending on the DIBMA/lipid ratio, the size of DIBMALPs can be broadly varied which makes them suitable for the incorporation of proteins of different sizes. Here, we examine the influence of the DIBMALP sizes and the presence of protein on the dynamics of encased lipids. As shown by a set of biophysical methods, the stability of DIBMALPs remains unaffected at different DIBMA/lipid ratios. Coarse-grained molecular dynamics simulations confirm the formation of viable DIBMALPs with an overall size of up to $35 \mathrm{~nm}$. Electron paramagnetic resonance spectroscopy of nitroxides located at the 5 th, 12th or 16th carbon atom positions in phosphatidylcholine-based spin labels reveals that the dynamics of enclosed lipids are not altered by the DIBMALP size. The presence of the membrane protein sensory rhodopsin II from Natronomonas pharaonis (NpSRII) results in a slight increase in the lipid dynamics compared to empty DIBMALPs. The light-induced photocycle shows full functionality of DIBMALPs-embedded NpSRII and a significant effect of the protein-to-lipid ratio during preparation on the NpSRII dynamics. This study indicates a possible expansion of the applicability of the DIBMALP technology on studies of membrane protein-protein interaction and oligomerization in a constraining environment.
\end{abstract}

Keywords: nitroxide spin label; electron paramagnetic resonance spectroscopy (EPR; ESR); polymer/lipid nanodiscs; phospholipid bilayer; membrane protein; negative-stain and cryo-transmission electron microscopy (EM); coarse-grained (CG) molecular dynamics (MD)

\section{Introduction}

Interactions of integral membrane proteins trigger a variety of processes in cellular membranes making important contributions to cell function and having a high pharmacological importance [1]. Application of various biophysical methods to the study of 
membrane protein structure and dynamics generally requires the preparation of watersoluble membrane protein samples in a native-like environment. Membrane proteins are usually studied in vitro by incorporating them into micelles, liposomes or nanolipoprotein particles (NLPs or nanodiscs) [2,3] with the help of detergents, which can negatively affect the stability and activity of proteins [4-6]. An alternative approach is to use amphiphilic styrene maleic acid (SMA) copolymers, which then form soluble SMA-lipid particles (SMALPs). SMA copolymers have been shown to solubilize membrane proteins by direct extraction from natural lipid bilayers or artificial membranes without applying detergents during any of the preparation steps ([7-10], reviewed, e.g., in [11-15]). The SMA copolymer-driven lipid solubilization also has the advantage of being non-selective concerning the lipid type $[16,17]$. The size of these nanoparticles is about $10 \mathrm{~nm}$ depending on the preparation routine [18]. The small size and single-particle character make SMALPs suitable for investigation of membrane proteins and their interactions [19] by spectroscopic techniques and also by electron microscopy (EM) and, in particular, by cryo-EM [20-23].

Recently, Keller and colleagues have shown that the diisobutylene/maleic acid (DIBMA) copolymers, which contain aliphatic diisobutylene styrene groups instead of aromatic ones, can, similar to SMA, solubilize lipids without using detergents resulting in the formation of stable DIBMA/lipid particles (DIBMALPs) somewhat larger than SMALPs [24,25]. Furthermore, the size of DIBMALPs can be varied in a broader range than that of SMALPs and monotonously decreases with an increasing polymer/lipid ratio [24,25]. Therefore, the DIBMA-based approach could be advantageous for the study of large membrane proteins or membrane protein complexes, as well as their interactions, by providing a higher degree of freedom for protein conformational changes or protein-protein interaction. Membrane proteins enclosed in stable polymer-stabilized lipid particles can be studied by different biophysical methods without loss of their structural and functional properties $[13,26,27]$.

Membrane proteins and their function could be influenced by their lipid surroundings [28-30]. Therefore, investigations of membrane proteins in a new lipid model system such as DIBMALPs require understanding of the properties of the DIBMA-enclosed lipid bilayer to confirm its biological relevance. However, it has remained unclear whether the resizing of DIBMALPs and the introduction of proteins can affect the enclosed lipid patch, thereby possibly influencing the structure, conformational stability, or dynamics of inserted proteins.

Dynamic properties of membrane proteins and lipid bilayers incorporated in liposomes, nanodiscs and SMALPs have been studied by electronic paramagnetic resonance (EPR) spectroscopy $[9,19,31-39]$. Upon introduction of spin probes into the proteins or lipids valuable structural and dynamic information were obtained [40-42]. Nitroxides attached to carbon atoms at different positions along the lipid acyl chain allow in-depth probing of the lipid bilayer in order to characterize the segmental chain mobility [42]; EPR combined with site-directed spin labeling reveals possible effects of confinement structure and dynamics of the encased proteins $[9,38]$. In addition, molecular dynamics (MD) simulations, including coarse-grained (CG) modeling, enable precise control over the content and simulated conditions of investigated systems and provide insights into the molecular mechanisms of lipid membrane solubilization and lipid nanoparticle stabilization by amphiphilic polymers [43]. For example, a recent CG MD simulation study examined the behavior of SMA copolymers with varying composition, charge and concentration in solution, as well as their interaction with lipid membranes [44].

In our previous work, we compared the dynamics of lipids in lipid nanoparticles formed by the SMA (3:1) and DIBMA copolymers using EPR spectroscopy and a set of phosphatidylcholine lipids with nitroxide groups introduced at different carbon atom positions [45]. Our results revealed that lipids are dynamically more constrained in SMALPs. Complimentary CG MD simulations indicated that, in the case of DIBMALPs, a single belt of polymer encircles lipids within the lipid nanoparticles and provided a possible 
explanation for the fact that lipid dynamics in DIBMALPs more closely resemble those in liposomes [45].

In the present work we focus on lipid and protein dynamics in DIBMALPs of different size prepared at different protein-to-lipid ratios. We apply EPR spectroscopy to study possible effects of varying sizes of DIBMALPs on the dynamics of enclosed lipids in the absence and presence of the membrane protein sensory rhodopsin II from Natronomonas pharaonis (NpSRII). To this end, we use a set of phosphatidylcholine lipids with nitroxide groups located at the 5th, 12th or 16th carbon atom positions along the hydrocarbon chain of the lipid and the saturated neutral lipid 2-dimyristoyl-sn-glycero-3-phosphocholine (DMPC). The EPR results show that neither the size of the nanoparticle nor the presence of the protein significantly alters the lipid dynamics in DIBMALPs. The light-induced photocycle of DIBMALP-embedded NpSRII reveals its full functionality, and the photocycle dependence on the protein-to-lipid ratio. Dynamic light scattering, electron microscopy and atomic force microscopy characterize the DIBMALP samples as homogeneous preparations of nanosized discoidal particles. Furthermore, CG MD simulations complement our experiments and show formation of stable DIBMALPs with an overall size up to $35 \mathrm{~nm}$ and uniform dynamics of the enclosed lipids.

\section{Results}

\subsection{Biophysical Characterization of Empty and Protein-Containing DIBMALPs}

The general scheme for the preparation of DIBMALP samples for further characterization is shown in Figure 1a. In a first step to characterize DIBMALPs of different sizes, we prepared DMPC liposomes and DMPC proteoliposomes containing NpSRII, and solubilized them with DIBMA copolymer at different DIBMA/DMPC weight ratios of 1:2, 1:1 or 2:1 (1:1 and 2:1 for the proteoliposomes) while keeping the lipid concentration constant (Figure 1a). At all selected DIBMA/lipid ratios, we could successfully obtain lipid nanoparticles.Dynamic light scattering (DLS) was employed to analyze the size distribution of particles in the preparations obtained upon lipid solubilization by the DIBMA copolymer. Figure $1 \mathrm{~b}$ shows the DLS data for DIBMA/DMPC particles with or without protein after mixing of DMPC liposomes with $4 \%(m / v)$ DIBMA at different DIBMA/DMPC weight ratios. All samples show the unimodal character of intensity-weighted size distributions indicating the presence of monodisperse nanosized particles (Figure 1b). The significant shift of the respective size distributions of empty and protein filled DIBMALPs shows that not only the DIBMA/DMPC weight ratio but also the presence of the protein affects the average size of the DIBMALPs.

Table 1 summarizes the DLS data and shows that the overall size of DIBMALPs gradually decreases with increasing DIBMA/DMPC ratio. The measured z-average size of DIBMALPs at a DIBMA/DMPC weight ratio of 1:2 is approximately $54 \mathrm{~nm}$. The size of particles decreases to around $42 \mathrm{~nm}$ at a DIBMA/DMPC weight ratio of 1:1 and decreases further to less than $30 \mathrm{~nm}$ at a DIBMA/DMPC weight ratio of 2:1. These results are consistent with previously published data $[24,25]$. The protein containing DIBMALPs are $\sim 30 \%$ smaller.

To further validate individual DIBMALPs shapes, we performed EM visualization of negatively stained particle samples prepared at 1:1 polymer-to-lipid weight ratio and deposited on glow-discharged 400-mesh copper grids. TEM-micrographs show the individual DIBMALPs as disc-shaped single particles (indicated by arrowheads) as well as stacked flat discoidal structures (indicated by arrows) (Figure 2a). The sizes of both flat discs and stacked discs at negative-stained EM micrographs correspond to the DLS data. Further visualization of assembled DIBMALPs by cryo-TEM provided images of single particles, both face-on and edge-on, but not of such stacked discs as on EM-micrographs of negatively stained samples (Figure 2a,b). Thus, the EM data confirm the proper disc shapes of DIBMALPs in our preparations. 


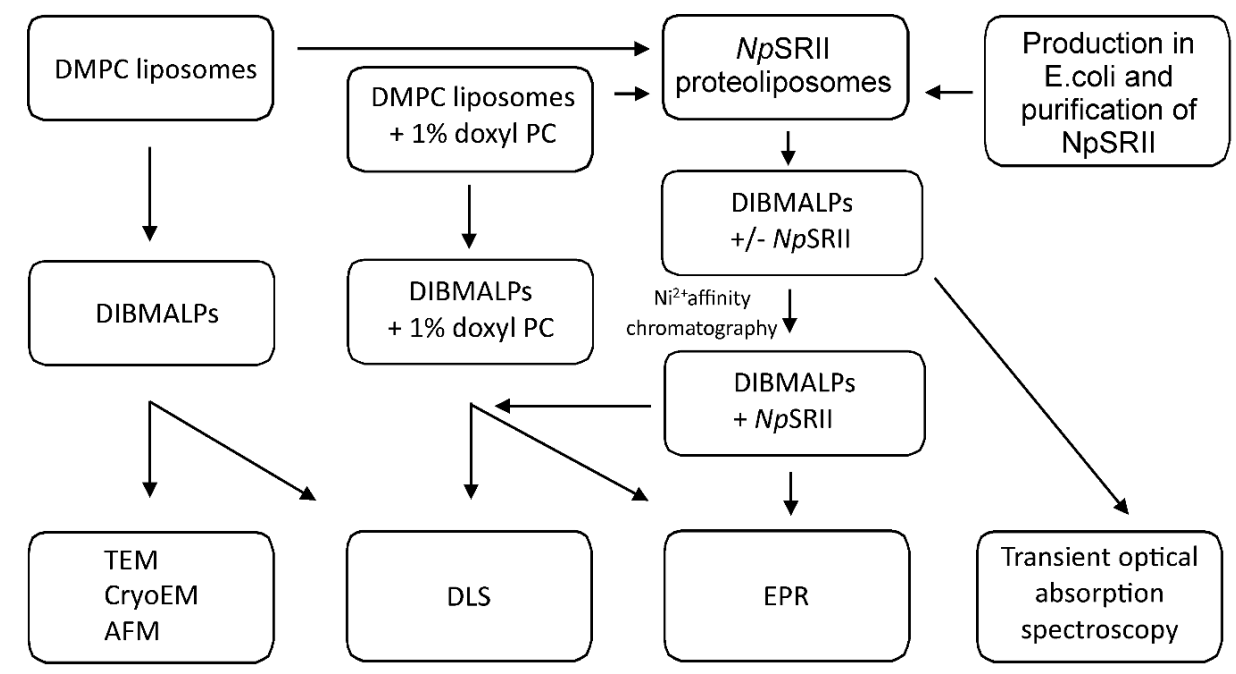

(a)

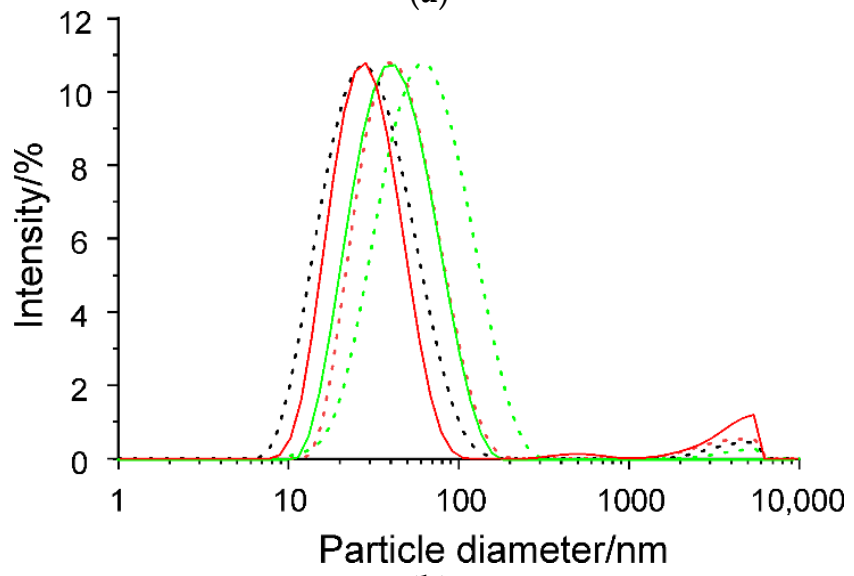

(b)

Figure 1. Characterization of lipid bilayer nanoparticles formed by diisobutylene/maleic acid (DIBMA). (a) Scheme showing the preparation of empty and protein-filled DIBMA lipid particles (DIBMALPs) and their biophysical characterization. (b) Representative dynamic light scattering (DLS) intensity-weighted size distributions of empty (broken lines) and NpSRII containing (continuous lines) DIBMALPs formed upon addition of $4 \%(\mathrm{~m} / \mathrm{v})$ DIBMA at a DIBMA/2-dimyristoyl-sn-glycero3-phosphocholine (DMPC) weight ratio of 1:2 (green traces), 1:1 (red traces) and 2:1 (black traces) to DMPC liposomes. All intensities were normalized to that of the black trace.

Table 1. $z$-Average diameter, $z$, of DIBMALPs determined by DLS at different DMPC/DIBMA ratios for preparations in absence and presence of NpSRII (average values of at least two repetitions). * In addition, the values from [45] are given for comparison, and were determined at a concentration (DIBMA:lipid) of 5:1.

\begin{tabular}{ccc}
\hline \multirow{2}{*}{ DIBMA/DMPC $w / w$} & \multicolumn{2}{c}{ Z-Average Hydrodynamic Diameter [nm] } \\
\cline { 2 - 3 } & - NpSRII & + Np SRII \\
\hline $1: 2$ & $53.9 \pm 0.4$ & $36.3 \pm 4.6$ \\
$1: 1$ & $41.5 \pm 2.0$ & $25.9 \pm 1.0$ \\
$2: 1$ & $26.5 \pm 1.6$ & - \\
$5: 1^{*}$ & $26.2 \pm 3.0$ & - \\
\hline
\end{tabular}




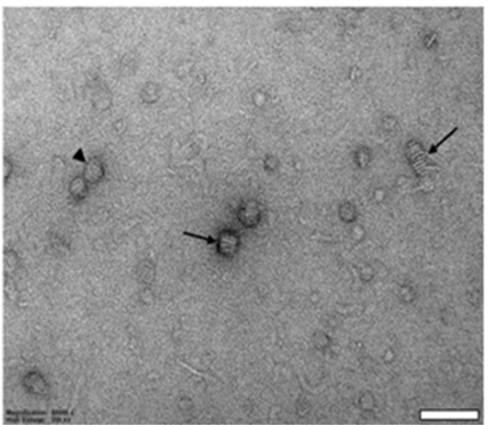

(a)

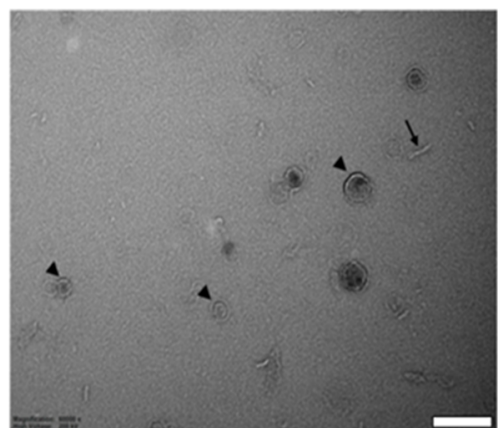

(b)

Figure 2. EM micrographs of DIBMA/DMPC nanoparticles. Negative-stain TEM (a) and cryo-TEM image (b) at 1:1 DIBMA/lipid weight ratio. The arrowheads and arrows indicate particles observed face-on and edge-on, respectively. Scale bar $100 \mathrm{~nm}$.

Atomic force microscopy (AFM) was used for profiling the surfaces of assembled DIBMALPs. Like EM micrographs, solution AFM images of DIBMALP preparations at 1:1 polymer-to-lipid weight ratio show individual nanoparticles (Figure 3a) as well as stacked disc-shaped structures (Figure 3c). At the tested particles, the height of DIBMALPs (in respect to the mica support) corresponds to the expected lipid bilayer thickness of about 3.0-4.0 nm. These data are consistent with our previously published AFM results obtained for SMALPs [46] and confirm that the DIBMA belt supports the typical lipidbilayer thickness.

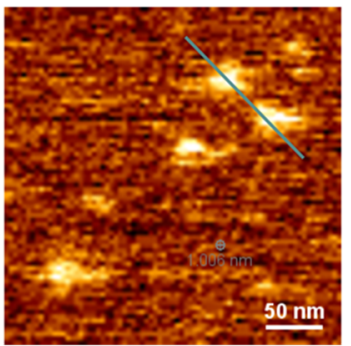

(a)

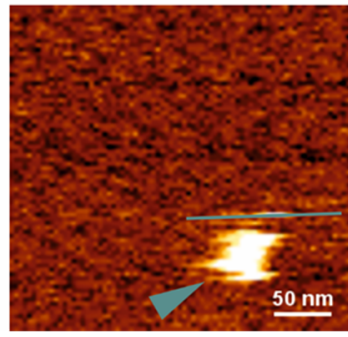

(c)
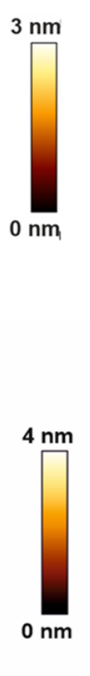

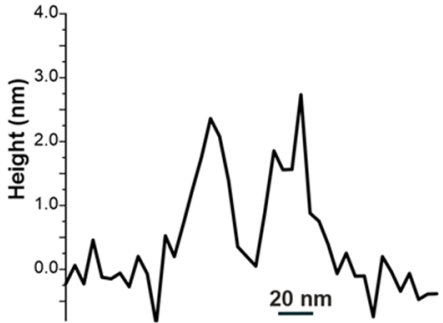

(b)

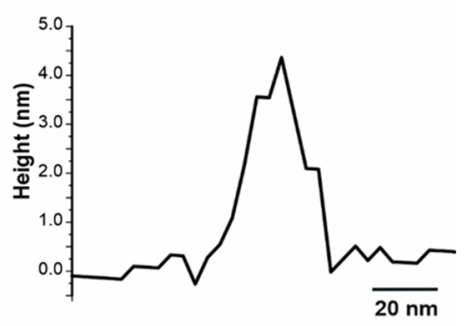

(d)

Figure 3. Atomic force microscopy (AFM) analysis of DIBMA/DMPC nanoparticles. (a,c) AFM images of DIBMA/DMPC nanoparticles prepared at 1:1 DIBMA/lipid weight ratio. The arrowhead indicates the presence of lipid nanoparticle stacks. (b,d) Height profile corresponding to the green line in the 2D image in $(\mathbf{a}, \mathbf{c})$ crossing individual lipid nanoparticles. The images are representative of at least two independent experiments.

\subsection{Lipid Dynamics and Ordering Are Not Affected by the Presence of NpSRII}

Using EPR spectroscopy we determined the dynamics of spin labeled lipids in DIBMALPs of different average sizes. The EPR line shape reflects the mobility of the nitroxide bound either to the 5th, 12th or 16th carbon atoms of the host phosphatidylcholine chain (in the following abbreviated as 5-doxyl PC, 12-doxyl PC and 16-doxyl PC) (Figure 4). Fast 
reorientational motion with rotational correlation times below $1 \mathrm{~ns}$ results in three equally spaced sharp lines of similar amplitude. Rotational correlation times in the ns time scale or moderate spatial restriction of the nitroxide reorientational motion are reflected in spectra with increased and different linewidths for the three resonance lines and increased apparent hyperfine splitting. Rotational correlation times above a few tens of ns or strong spatial restrictions result in so-called powder spectra. For all studied samples (Figure $4 \mathrm{~b}$, left panel), the EPR spectra show a gradual decrease of the linewidths and apparent hyperfine splitting when comparing 5-, 12- and 16-doxyl PC. The motional restriction is largest for 5-doxyl PC with the nitroxides located close to the lipid head groups and smallest for 16-doxyl PC with the nitroxide located close to the center of the bilayer. The superimposed spectra for the three different sizes of DIBMALPs prove only very small differences revealing that the lipid dynamics are not much influenced by the DIBMALP size (Figure $4 \mathrm{~b}$, left panel). The same is true when compared the lipid dynamics in DIBMALPs in presence and absence of NpSRII (Figure $4 \mathrm{~b}$, right panel). The spectra of 5-doxyl PC do not significantly differ beyond noise, whereas the spectra of 12-doxyl PC and 16-doxyl PC in protein filled DIBMALPs seem to show a slight increase of lipid dynamics compared to empty DIBMALs, as indicated by the slightly different shapes (amplitudes, widths) of the low field peaks (between 343.0 and $343.5 \mathrm{mT})$.

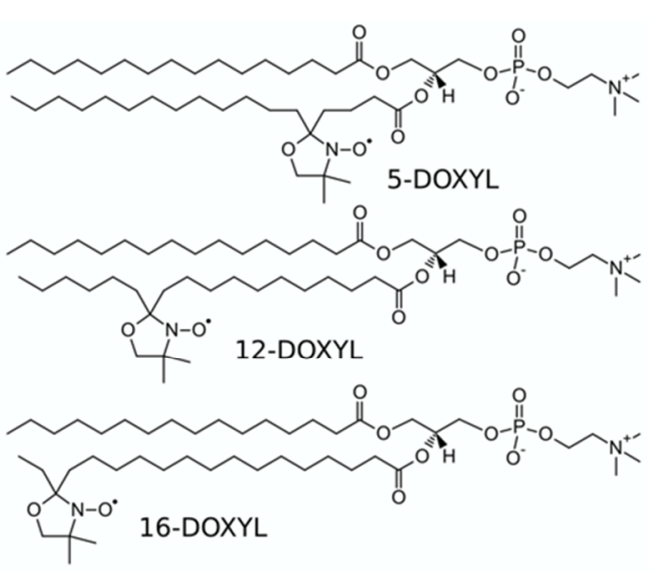

(a)

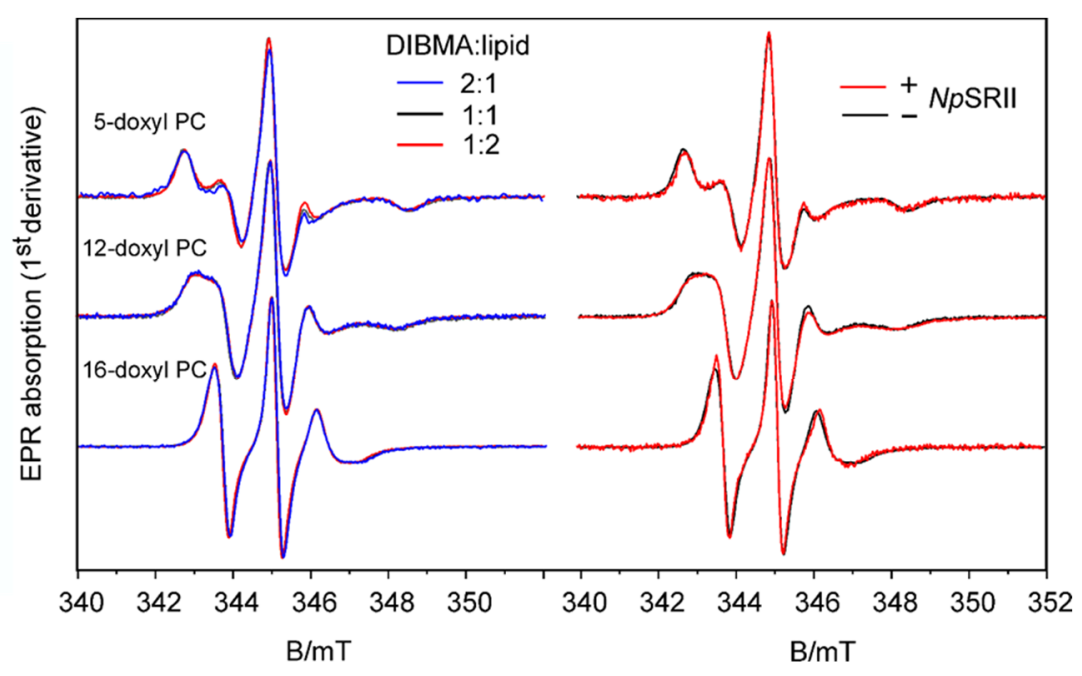

(b)

Figure 4. The dynamics of spin labeled lipids enclosed in DIBMALPs of different sizes and in absence and presence of NpSRII. (a) Doxyl-PCs with nitroxides bound at the 5th, 12th and 16th carbon position of the hydrocarbon chain. (b) EPR spectra recorded at room temperature for DIBMALPS prepared (left panel) with different DIBMA-to-lipid ratios ( $w / w), 2: 1$ (blue), 1:1 (black) and 1:2 (red), and (right panel) in presence (red) and absence (black) of NpSRII at a 1:1 (w/w) DIBMA-to-lipid ratio.

For a quantitative description, we performed spectra simulations based on a model of axial symmetric Brownian reorientation diffusion [47,48], according to Colbasevici et al. [45]. Fit parameters are the rotational correlation times, the inclination of the nitroxide axes with respect to the main lipid diffusion axes represented by the diffusion tilt angle, $\beta \mathrm{D}$, and the segmental order parameter, $\mathrm{S}$. The order parameter describes the motional restriction of the bound nitroxides, which results from the restoring potential acting on the lipids in the membrane. The values of these parameters are shown in Figure 5. A rigid crystal structure of the membrane is represented by the maximum value of the order parameter, $\mathrm{S}=1$, whereas membranes in a state of total dynamic disorder are characterized by the lowest possible value, $S=0$. For 5 -doxyl PC $S$ ranges between 0.59 and 0.66 , with the lower values found for DIBMALPs filled with NpSRII. The corresponding nitroxide position is located in the most ordered region of the bilayer, in agreement with earlier finding for 
the lipid dynamics in nanodiscs and liposomes [45]. The presence of NpSRII seems to slightly increase the local lipid disorder, whereas the rotational correlation time is not significantly affected by the size of the nanoparticle or the presence of the protein. The values of $S$ for 12-doxyl PC represent intermediate values. The rotational correlation times of the lipids in protein filled DIBMALPs are slightly smaller compared to those of empty DIBMALPs. 16-doxyl PC represents a location of the nitroxide close to the end of the lipid tail and thus in the middle of the bilayer. Its dynamics are less restricted compared to the 5 th and 12th positions, as reflected in a rotational correlation time at or below $1.6 \mathrm{~ns}$ and order parameters below 0.21 . The rotational correlation times do not differ for the present samples within their error margins. The behavior of the order parameter reveals a slightly decreased lipid ordering in the smallest DIBMALPs and in the presence of NpSRII.

Order parameter

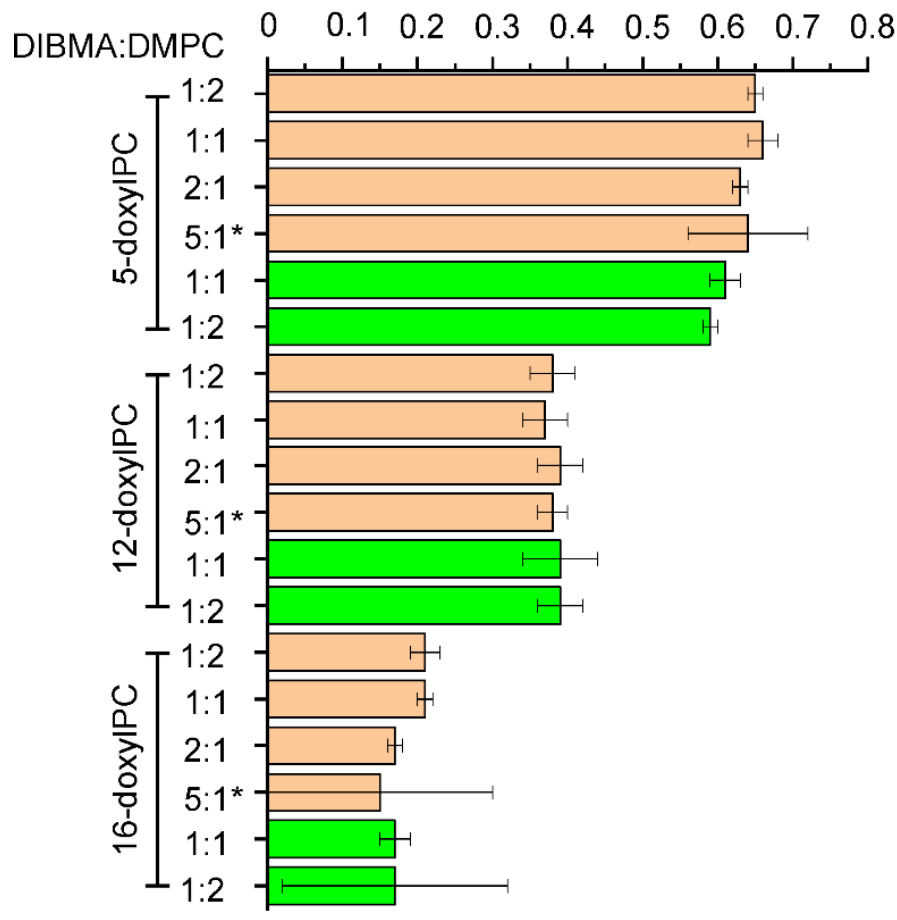

Rotational correlation time/ns

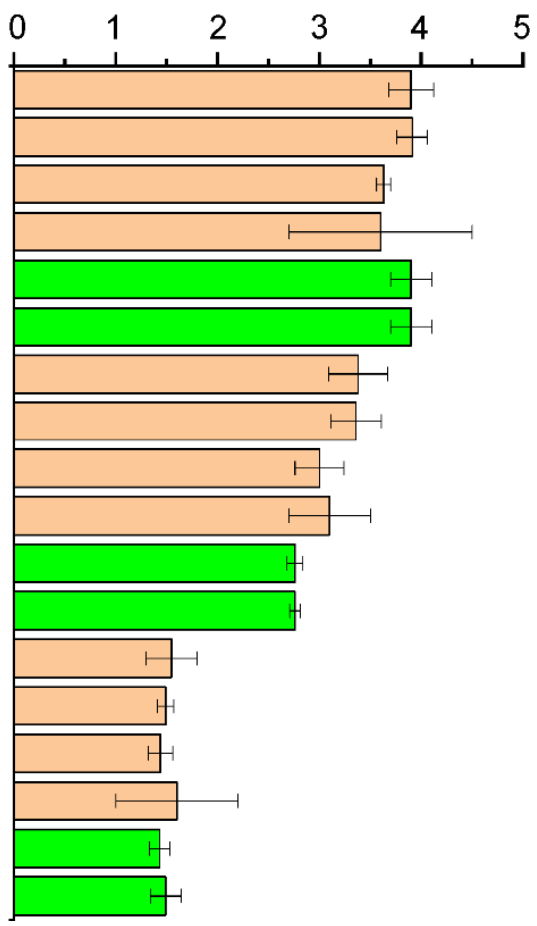

Figure 5. Order parameter $S$ and rotational correlation time $\tau$ calculated from the fits of the spectra shown in Figure 4 for empty (brown) and NpSRII containing (green) DIBMALPs. * For comparison, the values for empty DIBMALPs prepared with a concentration ratio of 5:1 are given (taken from [45]).

\subsection{Coarse-Grained Molecular Dynamics Simulations of Dibmalps Complement the Experimental} Results

In order to assess the overall viability of large DIBMALPs and the dynamics of lipids encased in them, we assembled coarse-grained molecular models of DIBMALPs ranging from approx. 30 to $50 \mathrm{~nm}$ in diameter, employing the same approach and DIBMA topology (Figure 6a,b) developed before for modeling SMALPs and smaller DIBMALPs (with the diameter of about $10 \mathrm{~nm}$ ) [45]. The obtained models were subjects for 1 microsecond long unconstrained MD simulations.

The simulations revealed that $30 \mathrm{~nm}$ and $35 \mathrm{~nm}$ DIBMALPs remain as planar discs throughout the whole simulation time (Figure $6 c, d$ ). The aspect ratio of their dimensions along the principal axes indicates that they also remain almost circular in shape (Figure 6i). The analysis of the acyl chain order parameters performed for DMPC in these DIBMALPs of different sizes (Figure 6j) do not show any difference in ordering of the lipid tails in full agreement with the EPR experiments. Also, the local bilayer thickness of both 30 and 
$35 \mathrm{~nm}$ DIBMALPs ( $3.3 \pm 0.7 \mathrm{~nm}$ and $3.2 \pm 0.8 \mathrm{~nm}$, respectively) match the experimental values obtained by means of AFM (see Figures 3 and $6 \mathrm{~g}, \mathrm{~h}$ ).

However, the behavior of the larger preformed DIBMALPs with the diameter of 40 and $50 \mathrm{~nm}$ during the unconstrained MD simulations was strikingly different to what we observed for 30 and $35 \mathrm{~nm}$ DIBMALPs. While the preformed discs are initially planar, they experience large undulations during the simulations and eventually bend into a cup-shaped structure (Figure 6e,f). The curved conformation of 40 and $50 \mathrm{~nm}$ DIBMALPs precluded the analysis of the order parameters in this case.

(a)

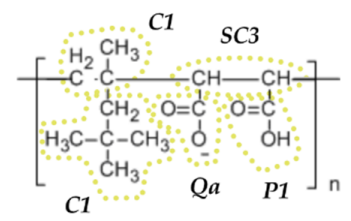

DIBMA

(c)
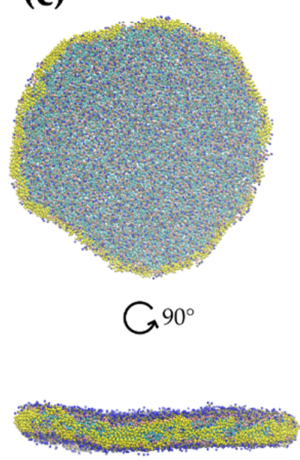

(g)

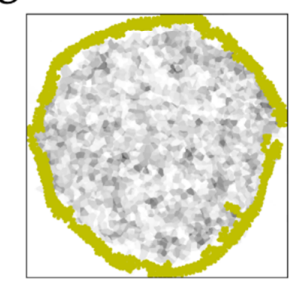

(d)
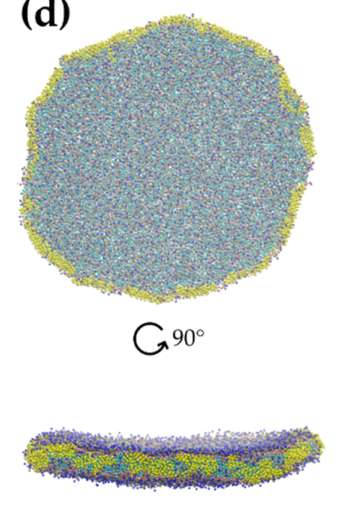

$\overline{10 \mathrm{~nm}}$

(h)

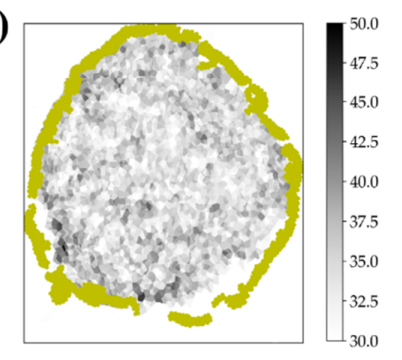

(b)

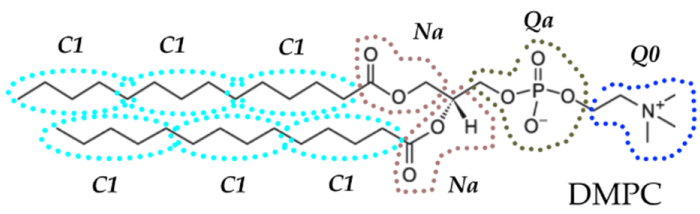

(e)
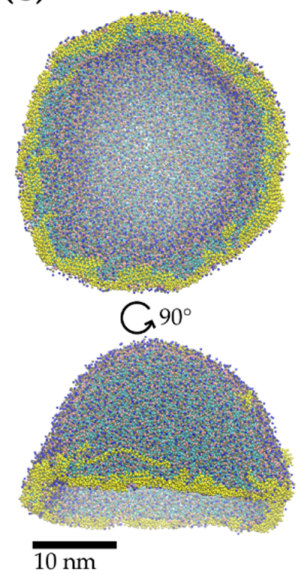

(i)

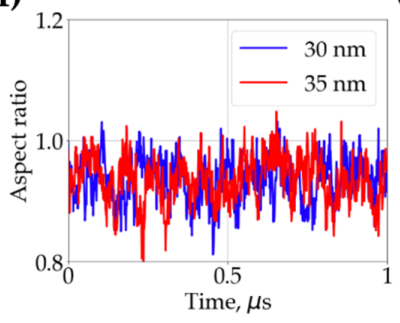

(f)

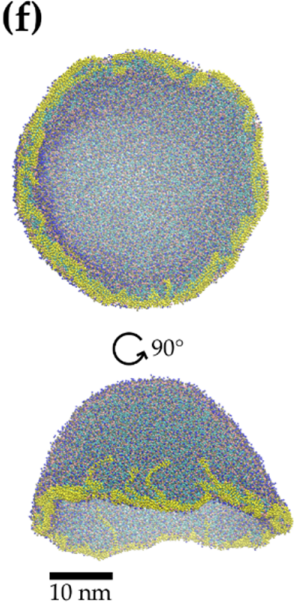

(j)

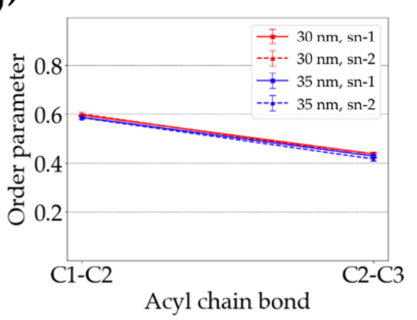

Figure 6. Coarse-grained (CG) molecular dynamics (MD) simulations of DIBMALPs of different diameter. The structural formulae of DIBMA copolymers (a) and DMPC lipids (b) used in this work. Periodically repeating chemical components of DIBMA polymers are enclosed in brackets and are marked with $\mathrm{n}$. The mapping of all-atom structures to CG MARTINI models is shown with the dotted contours captioned with the corresponding MARTINI CG particle types. The colors of CG particles correspond to those in panels (c-f). Final structures of $30 \mathrm{~nm}$ (c), $35 \mathrm{~nm}$ (d), $40 \mathrm{~nm}$ (e) and $50 \mathrm{~nm}$ (f) DIBMALPs after 1-microsecond-long unconstrained MD simulations. Local thickness of $30 \mathrm{~nm}$ (g) and $35 \mathrm{~nm}$ (h) DIBMALPs. Ratio of disc dimensions along the first two principal axes of the $30 \mathrm{~nm}$ and $35 \mathrm{~nm}$ DIBMALP as a function of the simulation time (i). Order parameters of the DMPC acyl chains in $30 \mathrm{~nm}$ and $35 \mathrm{~nm}$ DIBMALPs (j).

\subsection{The Photocycle of NpSRII in DIBMALPs Depends on the Protein-to-Lipid Ratio}

Sensory rhodopsin, NpSRII, an archaebacterial photoreceptor from Natronomonas pharaonis, belongs to the family of seven transmembrane helix proteins and is thus related to G-protein coupled receptors [49]. It mediates the photophobic response of $N$. pharaonis to green-blue light under aerobic conditions [50]. Activation by light leads to isomerization of the retinal chromophore, which is followed by a sequence of spectroscopically characterized intermediates, named $\mathrm{K}, \mathrm{L}, \mathrm{M}, \mathrm{N}$ and $\mathrm{O}$, leading back to the initial ground state 
(Figure 7a) [51]. The transition between the two M states ("switch") is accompanied by an outward movement of helix F [52,53], which was shown to trigger a conformational change of the associated transducer molecule. The photocycle kinetics and the corresponding conformational transitions of NpSRII are sensitive to changes of environmental conditions like lipid composition, temperature and $\mathrm{pH}[5,51,54]$. This is especially true for the transitions following the M-decay. Hence, these transitions are examined here to prove protein function and determine possible effects of protein-polymer or protein-protein interaction. Light-induced transient absorption changes were recorded for three characteristic wavelengths (Figure $7 \mathrm{~b}$ ). The decay of intermediate $\mathrm{M}$ was monitored at $400 \mathrm{~nm}$, the formation and decay of the O-state was followed at $550 \mathrm{~nm}$, and the recovery of the initial state was recorded at $500 \mathrm{~nm}$. The time constants from the multi-exponential fits $(0.25 \pm 0.01 \mathrm{~s}, 0.57 \pm 0.03 \mathrm{~s}, 1.2 \pm 0.1 \mathrm{~s}$ for protein/lipid ratio of 1:10) resemble those of $N p$ SRII reconstituted in purple membrane lipids $[38,55]$ and show that NpSRII retains its functionality in DIBMALPs. Strikingly, the increase of the weight lipid-to-protein ratio from 1:5 to 1:10 or 1:20 leads to a significant acceleration of the photocycle, most probably due to a decreased protein-protein interaction in DIBMALPs with increased lipid content (see Discussion).

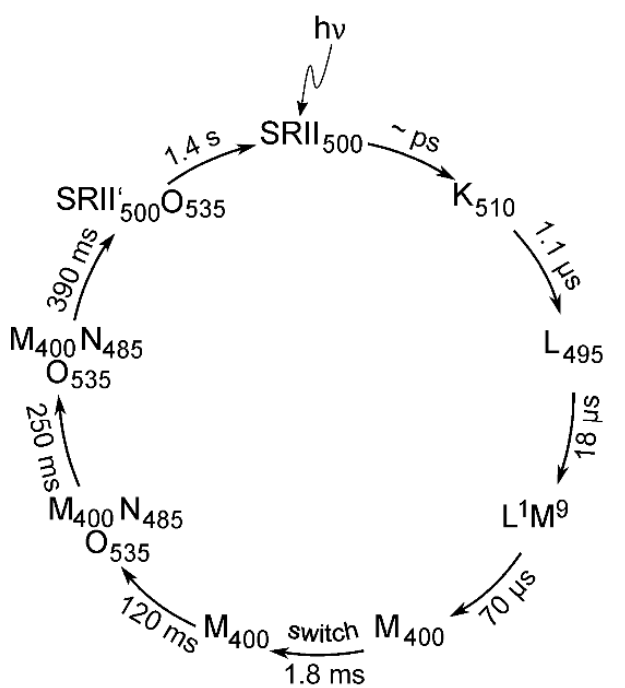

(a)

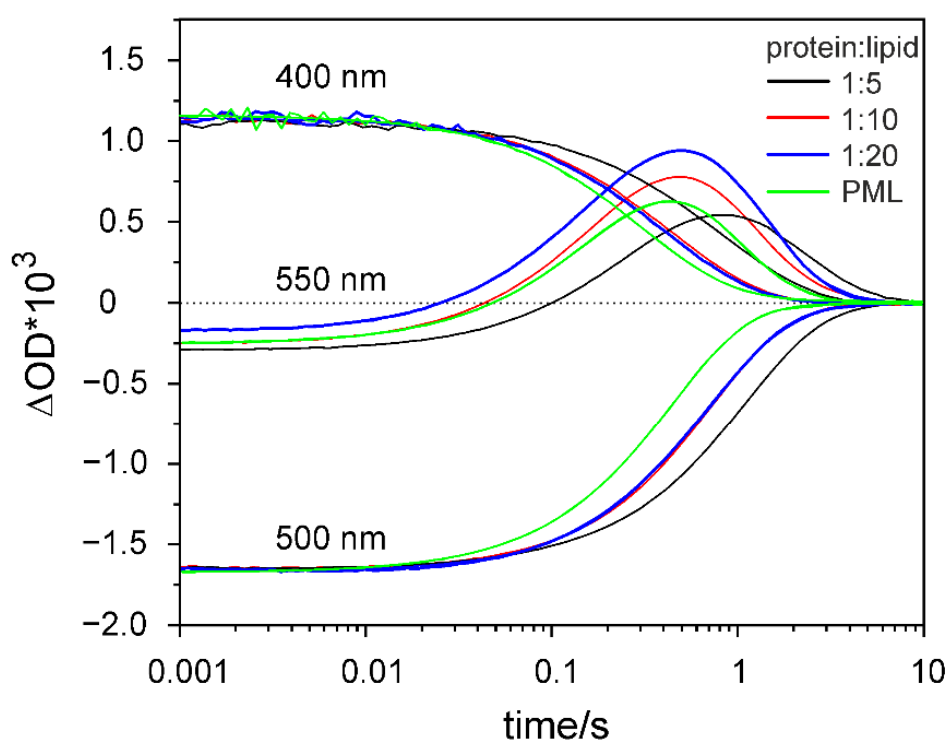

(b)

Figure 7. The photocycle of NpSRII in DIBMALPs depends on the protein-to-lipid ratio. (a) NpSRII photocycle characterized by transient changes in the optical absorption spectrum of the retinal chromophore. Subscripts indicate the wavelength of maximum absorption of the intermediates in $\mathrm{nm}$ (adapted from [51]). Time constants for the transitions between these intermediates are given according to the values for wildtype NpSRII reconstituted in purple membrane lipids [55]. (b) Transient optical absorption changes of NpSRII in DIBMALPs (DIBMA/lipid ratio 1:1) were recorded at $400 \mathrm{~nm}$ (Mintermediate), $500 \mathrm{~nm}$ (initial state) and $550 \mathrm{~nm}$ (O-state). The NpSRII-to-lipid ratio was varied, 1:5 (black), 1:10 (red) and 1:20 (blue). The fitted exponential curves are exemplarily shown for 1:20 as thin lines, which are indistinguishable from the measured transients. Maximum values of transients for 400 and $500 \mathrm{~nm}$ were normalized to the corresponding values of sample 1:20. For comparison, the traces of NpSRII in native purple membrane lipids (PML) are given (green) (data taken from [38]).

\section{Discussion}

In this work, we combine different biophysical approaches to compare the size distribution, particle homogeneity and lipid dynamics of DIBMALPs prepared at different DIBMA/DMPC weight ratios and in presence and absence of NpSRII. The DLS results show that an increase of the DIBMA content during the preparation leads to a corresponding 
decrease of the overall size of DIBMALPs for the tested DIBMA/DMPC compositions. These data are consistent with published values [24]; in this work, the authors observed DIBMA/DMPC particles with a hydrodynamic diameter of approximately $35 \mathrm{~nm}$ at a DIBMA/DMPC molar ratio of 0.08 and of approximately $18 \mathrm{~nm}$ at a DIBMA/DMPC molar ratio of 0.20 . Thus, our DLS data, in agreement with these previous results [24], show that varying weight ratios of DIBMA/DMPC controls the overall particle size in a range of about 30-50 nm. EM imaging represents individual DIBMALPs with sizes corresponding to the DLS values. Interestingly, TEM micrographs show the presence of stacked discs. These discs seem to be similar to those observed in negative-stained samples of phospholipid bilayers from purified plasma apolipoproteins and synthetic phospholipids. These structures have a regular repeat distance close to the thickness of a phospholipid bilayer [56,57]. Similar stacks have been observed also in nanodiscs [2]. Since negative staining can cause artefacts owing to fast dehydration of the sample [58], we combined negative staining with plunge-frozen samples in cryo-EM. Cryo-EM enables to avoid such artefacts because the rapid cryo-immobilization (plunge freezing used in this work) preserves the aqueous environment of the specimen whilst also preventing its possible damage [59]. We used AFM because of its high axial resolution that proved the native-like thickness of the assembled DIBMALPs (Figure 3), in agreement with previous data obtained for SMALPs [46].

The MD simulations confirm the viability and overall stability of planar DIBMALPs with the dimeter of 30 and $35 \mathrm{~nm}$, whereas larger DIBMALPs tend to curve and form cup-shaped structures during simulations (Figure 6e,f). Similar structures were previously shown to represent intermediates of simulated bilayer-to-vesicle $[60,61]$ and bicelle-tovesicle transitions [62], which encourages us to speculate that our simulations (restricted by 1 microsecond each due to the limited computational resources) would also end up in a vesicle-shaped conformation if continued. At the same time, the wrapping of DIBMAstabilized planar bilayers to cup-like structures was observed starting from a much larger number of lipids (more than 3000, see Table 2 in Methods) as compared to the plain lipid bilayers/bicelles where the bilayer-to-vesicle transition took place in systems consisting of as few as 512 lipids [60]. This process is supposed to be enthalpically unfavorable and entropically-driven; the conversion to vesicles decreases the solvent-accessible hydrophobic area of the bilayer [60]. Interactions between DIBMA and lipids may provide an additional enthalpic contribution and screen the hydrophobic edges of the bilayer. Both effects would stabilize the planar DIBMALPs. We are planning to investigate these effects systematically in future work.

Table 2. Overview of the simulated systems.

\begin{tabular}{cccc}
\hline $\begin{array}{c}\text { DIBMALP } \\
\text { Diameter, nm }\end{array}$ & Number of DMPC & Number of DIBMA & Box Size, nm $\times \mathbf{~ n m ~} \times \mathbf{n m}$ \\
\hline 30 & 2322 & 26 & $39.5 \times 39.5 \times 39.5$ \\
35 & 3162 & 30 & $45.7 \times 45.7 \times 45.7$ \\
40 & 4138 & 35 & $51.7 \times 51.7 \times 51.7$ \\
50 & 6520 & 44 & $60.8 \times 60.8 \times 60.8$ \\
\hline
\end{tabular}

Apart from the limitations of the utilized CG model [63], the apparent discrepancy in nanoparticle formation between the largest analyzed DIBMALPs (with DIBMA/lipid ratio 1:2) observed in the experiments and in our in silico simulations may be due to the different actual proportion of polymers and lipids in DIBMALPs, which is hard to control and follow. Another difference between the experimental and model systems was in the mechanism of DIBMALPs formation. While in the model system DIBMA molecules were added to a planar lipid bilayer, in the experiments, DIBMA molecules were added to spherical liposomes. Earlier we showed that copolymers extract whole lipid patches to form lipid nanoparticles [44]. If so, the DIBMA molecules would compete for lipids at higher DIBMA-to-lipid ratios. In addition, the average area of available free lipid patches would be smaller in case of proteoliposomes. Therefore, one could expect that the size of 
DIBMALPs would decrease with increased DIBMA-to-lipid and protein-to-lipid ratios, in agreement with experimental observations; see Figure $1 \mathrm{~b}$.

EPR spectroscopy shows that lipid dynamics does not depend on the size of the DIBMALPs in the studied size range in agreement with the results of the MD simulations. Thus, polymer-lipid interactions that might affect lipid dynamics at the polymer-lipid interface [45] play a negligible role. Similarly, the presence of NpSRII in DIBMALPs does not considerably change the average lipid dynamics. In contrast, different protein-to-lipid ratios in the preparation of NpSRII containing DIMALPs affect the photocycle kinetics. With 1600 lipids contained in a DIBMALP of $25 \mathrm{~nm}$ diameter (calculated from the values given in Table 2) and with the assumption that the treatment of the proteoliposomes with DIBMA does not change the protein-to-lipid ratio, the average number of NpSRII molecules encased in a single DIBMALP prepared with a protein-to-lipid ratio of 1:5 $(w / w)$ (corresponding to $1: 172(\mathrm{~mol} / \mathrm{mol}))$ is estimated to be nine or fewer. For a protein-to-lipid ratio of 1:20 the average occupation number is two, thereby reducing significantly possible protein-protein interaction or formation of oligomers. The photocycle of NpSRII is known to be modulated by protein-lipid interaction [5,38] and protein-protein interaction [64]. In particular, the interaction of $N p S R I I$ with its transducer $N p H$ trII accelerates the decay of $\mathrm{M}$ and $\mathrm{O}$ intermediates due to a more constraining NpSRII molecule, in which the conformations of $\mathrm{M}$ and $\mathrm{N}$ are destabilized [64]. NpSRII has been reported to form trimers [65]. It is thus tempting to speculate that the observed dependence of the photocycle on the lipid-to-protein ratio in the prepared DIBMALPs is due to a different degree of NpSRII trimerization or higher oligomerization. The present results thus indicate a possible expansion of the applicability of the DIBMALP technology for studies of membrane protein-protein interaction and oligomerization in a constrained environment.

\section{Materials and Methods}

\subsection{Chemicals}

1,2-Dimyristoyl-sn-glycero-3-phosphocholine (DMPC) was a kind gift from Lipoid (Ludwigshafen, Germany). 1-Palmitoyl-2-stearoyl-(5-doxyl)-sn-glycero-3-phosphocholine (16:0-5 doxyl PC), 1-palmitoyl-2-stearoyl-(12-doxyl)-sn-glycero-3-phosphocholine (16:0-12 doxyl PC), 1-palmitoyl-2-stearoyl-(16-doxyl)-sn-glycero-3-phosphocholine (16:0-16 doxyl PC) were bought from Sigma-Aldrich (St. Louis, MO, USA). DIBMA copolymer, commercially available under the trade name PureCube DIBMA 10 in TRIS (lyophilized in Tris-buffer, $\mathrm{pH}$ 7.5), was purchased from Cube Biotech (Monheim am Rhein, Germany). N-Dodecyl-B-D-maltoside (DDM) was acquired from Anatrace (Affymetrix, Cleveland, $\mathrm{OH}$, USA). Isopropyl- $\beta$-D-thiogalactopyranosid (IPTG) was bought from Carl Roth (Karlsruhe, Germany). EDTA-free complete protease inhibitor cocktail was procured from Roche Life Science (Mannheim, Germany). Ni-NTA superflow agarose was purchased from Qiagen (Hilden, Germany). All other reagents were of analytical grade.

Protein concentrations were determined using an UV-VIS spectrophotometer (UV2450, Shimadzu Corporation, Kyoto, Japan). The concentration of NpSRII was determined by using the known extinction coefficient of $50,000 \mathrm{M}^{-1} \mathrm{~cm}^{-1}$ at $\lambda=280 \mathrm{~nm}$.

\subsection{Protein Expression and Purification}

For purification purposes, NpSRII has a C-terminal 6xHis-tag. NpSRII-His was expressed in Escherichia coli BL21 (DE3) cells and purified according to [66,67] with minor modifications. Briefly, transformed cells were grown in Luria-Bertani (LB) medium containing $50 \mathrm{mg} / \mathrm{mL}$ kanamycin at $37^{\circ} \mathrm{C}$ to an optical density $\mathrm{OD}_{580}$ of 1.0. The overexpression of the protein was induced by addition of IPTG to a final concentration of $0.5 \mathrm{mM} .10 \mu \mathrm{M}$ all-trans retinal (Sigma, St. Louis, MO, USA) was also added. After an induction period of $3 \mathrm{~h}$ at $37^{\circ} \mathrm{C}$, cells were harvested $\left(4200 \times g ; 15 \mathrm{~min} ; 4^{\circ} \mathrm{C}\right)$, washed and resuspended $(1 / 100$ culture volume) in $150 \mathrm{mM} \mathrm{NaCl}, 25 \mathrm{mM} \mathrm{NaPi}$ (pH 8.0), 2 mM EDTA buffer containing a protease inhibitor mix. Cells were disrupted by sonication (Branson Sonifier II W-250, Heinemann, Germany). Membranes were isolated by centrifugation $\left(50,000 \times g ; 1 \mathrm{~h} ; 4^{\circ} \mathrm{C}\right)$, 
and solubilized in buffer A (300 mM NaCl, $50 \mathrm{mM} \mathrm{NaPi}(\mathrm{pH} 8.0), 2 \%(w / v)$ DDM) overnight at $4{ }^{\circ} \mathrm{C}$. Solubilized membrane proteins were isolated by centrifugation $(50,000 \times g ; 1 \mathrm{~h}$; $4{ }^{\circ} \mathrm{C}$ ) followed by chromatography using Ni-NTA superflow material which was preequilibrated with buffer B $\left(300 \mathrm{mM} \mathrm{NaCl}, 50 \mathrm{mM} \mathrm{NaPi}\left(\mathrm{Na}_{2} \mathrm{HPO}_{4} / \mathrm{NaH}_{2} \mathrm{PO}_{4}\right) \mathrm{pH}\right.$ 8.0, $0.05 \%(w / v)$ DDM). Non-specifically bound proteins were removed by washing extensively with buffer B containing $30 \mathrm{mM}$ imidazole. His-tagged protein was eluted with buffer B containing $200 \mathrm{mM}$ imidazole. Fractions containing the desired protein were pooled and dialyzed against buffer $\mathrm{C}(500 \mathrm{mM} \mathrm{NaCl}, 10 \mathrm{mM}$ Tris $(\mathrm{pH} 8.0), 0.05 \%(w / v) \mathrm{DDM}))$ to remove imidazole. If not used directly for reconstitution, protein samples were flash frozen and stored at $-80{ }^{\circ} \mathrm{C}$.

\subsection{Liposome Preparations}

Powdered DMPC was dissolved in chloroform to a final lipid concentration of $7.37 \mathrm{mM}$ and if desired mixed with $1 \mathrm{~mol} \%$ of lipid spin label. Chloroform was evaporated under a stream of nitrogen gas. The resulting lipid film was dried under vacuum for at least $2 \mathrm{~h}$. The dried lipids were suspended in $50 \mathrm{mM}$ Tris (pH 7.5), $200 \mathrm{mM} \mathrm{NaCl}$ (buffer D) and vortexed. Subsequently, the multilamellar liposome suspension underwent five freeze-thaw cycles $\left(\mathrm{N}_{2} /\right.$ water bath at $\left.37^{\circ} \mathrm{C}\right)$, and, if not used directly, was stored in aliquots at $-80{ }^{\circ} \mathrm{C}$. Before reconstitution, the suspension of liposomes was extruded 31 times through polycarbonate membranes of $100 \mathrm{~nm}$ pore size using a Mini-Extruder Set (Avanti Polar Lipids, Alabaster, AL, USA) to get unilamellar vesicles.

\subsection{Proteoliposome Preparation}

Proteoliposomes were prepared as published before [29] with minor modifications. Briefly, preformed liposomes were equilibrated by stirring for $3 \mathrm{~h}$ with $\mathrm{n}$-dodecyl- $\beta$-Dmaltopyranoside (DDM) at room temperature with a lipid to detergent mixing ratio of 1:1 ( $\mathrm{mol} / \mathrm{mol})$. Before adding the protein solution, the lipid-detergent mixture was placed in an ultrasonic bath at room temperature for $10 \mathrm{~min}$. Then NpSRII was mixed with the equilibrated with DDM liposomes at a protein-to-lipid weight ratio of either 1:5, 1:10 or 1:20 $(w / w)$ and incubated for $30 \mathrm{~min}$ at room temperature with gentle shaking. To remove the detergent, the reconstitution of the NpSRII in liposomes was carried out using hydrophobic polystyrene beads, SM-2 Bio-Beads (Bio-Rad Laboratories, Munich, Germany). These hydrophobic polystyrene beads were washed thoroughly beforehand with methanol and water followed by buffer $\mathrm{D}$. The Bio-Beads were added to the protein-lipid detergent solution at a ratio of 10:1 (w/w) of the wet Bio-Beads to detergent. The samples were incubated for one hour at room temperature. A new portion of Bio-Beads was then added, and the samples were incubated at $4{ }^{\circ} \mathrm{C}$ overnight with gentle shaking. After removing the Bio-Beads, the proteoliposomes were collected by centrifugation $\left(15,800 \times g ; 30 \mathrm{~min} ; 4^{\circ} \mathrm{C}\right)$. Finally, they were resuspended in buffer A to a final lipid concentration of $7.4 \mathrm{mM}$.

\subsection{Preparation of DIBMALPS}

DIBMALPs were prepared as published before [13]. Briefly, to form DIBMA/lipid particles, a $4 \%(w / v)$ aqueous solution of DIBMA copolymer was added dropwise to the liposome suspension to get a copolymer/lipid weight ratio of 1:2, 1:1 or 2:1 at a final lipid concentration of $0.2 \%$ (3 mM) (a copolymer/lipid molar ratio of $0.033 ; 0.067 ; 0.133$, corresponding to a final polymer concentration of $0.1 \%, 0.2 \%$ and $0.4 \%(w / v)$, respectively). The assembly mixture was allowed to equilibrate for $1 \mathrm{~h}$ at room temperature and then for $16 \mathrm{~h}$ at $4{ }^{\circ} \mathrm{C}$. The resulting samples were centrifuged $\left(126,000 \times g ; 30 \mathrm{~min} ; 4^{\circ} \mathrm{C}\right)$ to remove aggregates. If necessary, the samples were concentrated using $3 \mathrm{kDa}$ MWCO Vivaspin 500 ultrafiltration devices (Sartorius, Epsom, UK).

\subsection{Preparation of DIBMALPs Containing NpSRII}

To form DIBMALPs containing NpSRII (in the following abbreviated as NpSRIIDIBMALPs), a $4 \%(w / v)$ aqueous solution of the DIBMA copolymer was added dropwise 
to the suspension of proteoliposomes to obtain a final copolymer/lipid weight ratio of 1:1 or $2: 1$. The mixture was then equilibrated for $1 \mathrm{~h}$ at room temperature and then at $4{ }^{\circ} \mathrm{C}$ for $16 \mathrm{~h}$. The samples were centrifuged $\left(126,000 \times g ; 30 \mathrm{~min} ; 4^{\circ} \mathrm{C}\right)$ to remove the unsolubilized proteins. The resulting samples were then incubated for one hour at room temperature with gentle agitation with $\mathrm{Ni}^{2+}-\mathrm{NTA}$ agarose (Qiagen), which had previously been equilibrated with buffer E (300 mM NaCl, $50 \mathrm{mM} \mathrm{NaPi}\left(\mathrm{Na}_{2} \mathrm{HPO}_{4} / \mathrm{NaH}_{2} \mathrm{PO}_{4}\right)$, pH 8.0). Atypically bound material was removed by washing with $40 \mathrm{mM}$ imidazole in buffer E. DIBMALPs containing His-tagged NpSRII were eluted with $200 \mathrm{mM}$ imidazole in buffer E and if necessary dialyzed against buffer $\mathrm{D}$. At both 1:1 and 2:1 copolymer/lipid weight ratios, the yield of affinity purified NpSRII after extracting from proteoliposomes was slightly more than $20 \%$.

\subsection{Dynamic Light Scattering}

DLS measurements were performed on a Zetasizer Nano ZS (Malvern Instruments, Worcestershire, UK) at $550 \mathrm{~nm}$ and $25{ }^{\circ} \mathrm{C}$. Data represent the average of three sets of 14 runs of $10 \mathrm{~s}$ each. The particle size distribution was obtained by using the ZETASIZER software package Ver. 7.02. under the assumption that DIBMA/lipid nanoparticles were spherically shaped.

\subsection{Transmission Electron Microscopy}

Freshly prepared DIBMALPs (2 mg lipid/mL) were diluted 1:100 in buffer $\mathrm{D}$, and $4 \mu \mathrm{L}$ were applied onto negatively glow-discharged carbon-coated 400-mesh copper grids (300-mesh formvar/carbon-coated) (Ted Pella, Redding, CA, USA) for $1 \mathrm{~min}$. Excess liquid was removed by blotting with filter paper. The grid was washed twice shortly with buffer and stained 4 min with $2 \%$ uranyl acetate and blotted. Digital micrographs were collected using a JEM2100Plus Transmission Electron Microscope (JEOL, Akishima City, Japan) operated at $200 \mathrm{kV}$ equipped with a XAROSA CMOS 20 Megapixel Camera (EMSIS GmbH, Muenster, Germany).

\subsection{Cryo-Transmission Electron Microscopy}

DIBMALPs (2 mg lipid/mL) were diluted 1:10 in buffer D and $4 \mu \mathrm{L}$ of DMPC/DIBMA suspension was placed on glow-discharged holey copper grid (Quantifoil R2/2 200 Mesh). Grids were blotted for $3 \mathrm{~s}$ at $10{ }^{\circ} \mathrm{C}, 80 \%$ humidity, and frozen in liquid ethane using EM GP2 plunge freezer (Leica, Wetzlar, Germany). Grids were transferred into a JEOL JEM2100Plus Transmission Electron Microscope operating at $200 \mathrm{kV}$. Digital micrographs were recorded manually with a XAROSA CMOS 20 Megapixel Camera.

\subsection{Atomic Force Microscopy}

For AFM imaging, $250 \mu \mathrm{L}$ of a solution of DIBMALPs was put in contact with freshly cleaved mica, previously glued to a coverslip, and diluted with additional $250 \mu \mathrm{L}$ of buffer (50 mM Tris, $200 \mathrm{mM} \mathrm{NaCl}, \mathrm{pH}$ 7.5). The DIBMALP sample was incubated for $10 \mathrm{~min}$, then mica was washed 3 times with $500 \mu \mathrm{L}$ of the buffer and then allowed to equilibrate at room temperature before analysis. DIBMALPs were imaged using a JPK NanoWizard II system (JPK Instruments, Berlin, Germany) mounted on an Olympus IX71 Inverted Microscope (Olympus Corporation, Tokyo, Japan). Intermittent contact (IC or tapping) mode images were taken using an SNL-10 V-shaped silicon nitride cantilevers (Bruker AFM Probes, Camarillo, CA, USA) with a typical spring constant of $0.06 \mathrm{~N} / \mathrm{m}$. The cantilever oscillation was tuned to a frequency between 3 and $10 \mathrm{kHz}$, and the amplitude was set between 0.3 and $0.7 \mathrm{~V}$. The amplitude was varied during the experiment to minimize the force of the tip on the particles. The scan rate was set to $0.3-0.7 \mathrm{~Hz}$. Images were processed by the JPK processing software, applying a smoothing function. Lipid nanoparticle thickness was measured based on the height profiles from the mica (taken as $0 \mathrm{~nm}$ ). 


\subsection{Electron Paramagnetic Resonance Spectroscopy}

EPR measurements were performed as described previously [68]. Briefly, room temperature continuous wave (cw) EPR spectra were recorded on a home-built EPR spectrometer equipped with a dielectric resonator (Bruker Biospin, Ettlingen, Germany). Glass capillaries of $0.9 \mathrm{~mm}$ inner diameter were filled with sample volumes of $\sim 20 \mu \mathrm{L}$. The measurements were recorded at $(9.686 \pm 0.005) \mathrm{GHz}$ in a B-field range from $340 \mathrm{mT}$ to $352 \mathrm{mT}$. The field modulation was set to $0.22 \mathrm{mT}$. The temperature during the measurements was $295 \mathrm{~K}$.

\subsection{EPR Spectra Simulations}

Other variables, which were allowed to vary during the fittings, were the rotational diffusion tensor of axial symmetry defined by $R_{\perp}$ and $R_{\|}$, the coefficient of the orienting (restoring) potential, $\mathrm{C}_{20}$, from which MultiComponent calculates the order parameter $\mathrm{S}$, and an isotropic linewidth, $\mathrm{W}$. The errors were determined by repeating the fits with different starting parameters of the angle $\beta_{D}$. For this purpose, the value of $\beta_{D}$ was changed by $\pm 5^{\circ}$ around the best-fit value.

\subsection{Transient Optical Absorption Spectroscopy}

Transient optical absorption experiments were carried out as described previously [55]. A $50 \mathrm{~W}$ halogen lamp with an infrared cutoff filter (KG-2) and $400 \mathrm{~nm}, 500 \mathrm{~nm}$ or $550 \mathrm{~nm}$ interference filters illuminated the sample-filled quartz cuvette inside a sample holder, which was temperature-controlled to $298 \mathrm{~K}$. The transmitted light was passed through a second interference filter and detected by a photodiode. A flashlight with a flash duration of $\sim 80 \mu \mathrm{s}$ equipped with a $475 \mathrm{~nm}$ edge filter provided excitation perpendicular to the transmission beam. The amplified signal was recorded with an analog-to-digital converter connected to a standard PC. For the transitions between the late photocycle intermediates ( $t>2 \mathrm{~ms}$ ) studied here, the kinetics determined by flashlight excitation were indistinguishable from those determined by pulsed laser excitation [68]. The transient absorption changes were recorded on samples buffered in $50 \mathrm{mM}$ Tris, $\mathrm{pH} 7.50,200 \mathrm{mM} \mathrm{NaCl}$ at concentrations of approximately $5 \mu \mathrm{M}$.

\subsection{Molecular Dynamics Simulations}

In order to simulate large DIBMALPs, we used the coarse-grained model of the DIBMA polymer (consisting of 52 units with the 1:1 diisobutylene/maleic acid ratio) developed by us recently [45] within the framework of the popular MARTINI force field [63]. The standard MARTINI library contains the CG force field parameters for DMPC.

We used Gromacs 2018.1 for all MD simulations [69]. The DIBMALPs of various sizes (see Table 2 for the overview of all simulated systems) were assembled starting from a circular DMPC lipid patch created by the insane.py script [70] surrounded by DIBMA polymers in the amount sufficient to form a single layer of polymers at the rim of the lipid patch (estimated from the contour length of a single polymer and the lipid patch perimeter).

Energy minimization using the steepest descent algorithm prefaced each equilibration simulation run in the NVT ensemble (simulation time equaled $1 \mu \mathrm{s}$ ) maintained by the V-rescale thermostat $\left(\mathrm{T}=320 \mathrm{~K}, \tau_{\mathrm{t}}=1.0 \mathrm{ps}\right)$ during which the phosphate groups of lipids were constrained in the plane perpendicular to the normal of the lipid patch using a harmonic potential with the force constant $=1000 \mathrm{~kJ} / \mathrm{mol} / \mathrm{nm}^{2}$.

The production simulations were run for $1 \mu \mathrm{s}$ each in the NPT ensemble using the same thermostat and the Parrinello-Rahman barostat (time constant $=12.0 \mathrm{ps}$, compressibility $=$ $3 \times 10^{-4} \mathrm{bar}^{-1}$, as recommended in [71], applied isotropically). All CG simulations were performed in the explicit solvent with the standard water model, at $0.15 \mathrm{M}$ concentration of $\mathrm{NaCl}$. The reaction field approach was used to treat the long-range electrostatics with the relative permittivity of 15 and the Coulomb cut-off of $1.1 \mathrm{~nm}$ [71]. A time step of $20 \mathrm{fs}$ was utilized for all simulations. The Verlet pair-lists cutoff scheme was used and the neighbor list was updated every 20 steps. Periodic boundary conditions were applied in all simulations. 
For analysis, the DIBMALPs were aligned such that their center-of-weight motion was removed, and their principal axes were aligned with the coordinate axes at every trajectory step. In-house Python scripts exploiting MDAnalysis [72] were used for the alignment, calculation of the aspect ratio along the first principal axes and analysis of the order parameters of lipid acyl chains. Local thickness was calculated as in [73].

Author Contributions: Conceptualization, N.V., K.C. and H.-J.S.; methodology, N.V., K.C., O.-E.P., A.Y.M. and H.-J.S.; software, P.O., M.B., K.C. and O.-E.P.; validation, N.V., P.O., M.B., F.K., M.R. and M.S.; formal analysis, N.V., K.V.S. and A.Y.M.; investigation, N.V., M.B., F.K., M.R., M.S., A.B.-G., B.B. and H.-J.S.; resources, O.-E.P., K.C., K.V.S. and H.-J.S.; data curation, N.V. and H.-J.S.; writingoriginal draft preparation, all authors; writing—review and editing, N.V., K.C., P.O., M.B. and H.-J.S.; visualization, P.O., M.B., O.-E.P. and H.-J.S.; supervision, K.V.S. and H.-J.S.; project administration, H.-J.S. and K.V.S.; funding acquisition, H.-J.S. and K.V.S. All authors have read and agreed to the published version of the manuscript.

Funding: This research was funded by German Research Foundation (DFG, STE640/15) to H.-J.S., (DFG, SFB 944/3-2020, TP 26) to K.C., and RFBR grant no. 18-504-12045 to K.V.S., A.Y.M., K.V.S. and H.-J.S. would like to thank the German Academic Exchange Service (DAAD) and its Ostpartnerschaftenprogramm for support. The authors acknowledge the support from Deutsche Forschungsgemeinschaft (DFG) via the Open Access Publishing Fund of Osnabrück University.

Institutional Review Board Statement: Not applicable.

Informed Consent Statement: Not applicable.

Data Availability Statement: All data generated or analyzed during this study are included in this published article.

Acknowledgments: The TEM and AFM imaging was performed in the electron microscopy and optical imaging unit of Integrated Bioimaging Facility (iBiOs) at the Center of Cellular Nanoanalytics (CellNanOs), University of Osnabrück.

Conflicts of Interest: The authors declare no conflict of interest. The funders had no role in the design of the study; in the collection, analyses, or interpretation of data; in the writing of the manuscript, or in the decision to publish the results.

\section{References}

1. Overington, J.P.; Al-Lazikani, B.; Hopkins, A.L. How many drug targets are there? Nat. Rev. Drug Discov. 2006, 5, 993-996. [CrossRef]

2. Bayburt, T.H.; Sligar, S.G. Self-assembly of single integral membrane proteins into soluble nanoscale phospholipid bilayers. Protein Sci. 2003, 12, 2476-2481. [CrossRef]

3. Denisov, I.G.; Sligar, S.G. Nanodiscs in Membrane Biochemistry and Biophysics. Chem. Rev. 2017, 117, 4669-4713. [CrossRef] [PubMed]

4. De Zorzi, R.; Mi, W.; Liao, M.; Walz, T. Single-particle electron microscopy in the study of membrane protein structure. Microscopy 2016, 65, 81-96. [CrossRef] [PubMed]

5. Klare, J.P.; Bordignon, E.; Doebber, M.; Fitter, J.; Kriegsmann, J.; Chizhov, I.; Steinhoff, H.J.; Engelhard, M. Effects of solubilization on the structure and function of the sensory rhodopsin II/transducer complex. J. Mol. Biol. 2006, 356, 1207-1221. [CrossRef] [PubMed]

6. Zoonens, M.; Comer, J.; Masscheleyn, S.; Pebay-Peyroula, E.; Chipot, C.; Miroux, B.; Dehez, F. Dangerous liaisons between detergents and membrane proteins. The case of mitochondrial uncoupling protein 2. J. Am. Chem. Soc. 2013, 135, 15174-15182 [CrossRef]

7. Knowles, T.J.; Finka, R.; Smith, C.; Lin, Y.P.; Dafforn, T.; Overduin, M. Membrane proteins solubilized intact in lipid containing nanoparticles bounded by styrene maleic acid copolymer. J. Am. Chem. Soc. 2009, 131, 7484-7485. [CrossRef] [PubMed]

8. Jamshad, M.; Lin, Y.P.; Knowles, T.J.; Parslow, R.A.; Harris, C.; Wheatley, M.; Poyner, D.R.; Bill, R.M.; Thomas, O.R.; Overduin, M.; et al. Surfactant-free purification of membrane proteins with intact native membrane environment. Biochem. Soc. Trans. 2011, 39, 813-818. [CrossRef] [PubMed]

9. Orwick-Rydmark, M.; Lovett, J.E.; Graziadei, A.; Lindholm, L.; Hicks, M.R.; Watts, A. Detergent-free incorporation of a seventransmembrane receptor protein into nanosized bilayer Lipodisq particles for functional and biophysical studies. Nano Lett. 2012, 12, 4687-4692. [CrossRef] 
10. Orwick, M.C.; Judge, P.J.; Procek, J.; Lindholm, L.; Graziadei, A.; Engel, A.; Grobner, G.; Watts, A. Detergent-free formation and physicochemical characterization of nanosized lipid-polymer complexes: Lipodisq. Angew. Chem. Int. Ed. 2012, 51, $4653-4657$. [CrossRef] [PubMed]

11. Dorr, J.M.; Scheidelaar, S.; Koorengevel, M.C.; Dominguez, J.J.; Schafer, M.; Van Walree, C.A.; Killian, J.A. The styrene-maleic acid copolymer: A versatile tool in membrane research. Eur. Biophys. J. 2016, 45, 3-21. [CrossRef] [PubMed]

12. Lee, S.C.; Pollock, N.L. Membrane proteins: Is the future disc shaped? Biochem. Soc. Trans. 2016, 44, 1011-1018. [CrossRef] [PubMed]

13. Overduin, M.; Esmaili, M. Structures and Interactions of Transmembrane Targets in Native Nanodiscs. SLAS Discov. 2019, 24, 943-952. [CrossRef]

14. Ravula, T.; Hardin, N.Z.; Ramamoorthy, A. Polymer nanodiscs: Advantages and limitations. Chem. Phys. Lipids 2019, 219, 45-49. [CrossRef] [PubMed]

15. Lemieux, M.J.; Overduin, M. Structure and function of proteins in membranes and nanodiscs. Biochim. Biophys. Acta Biomembr. 2020, 1863, 183445. [CrossRef] [PubMed]

16. Arenas, R.C.; Klingler, J.; Vargas, C.; Keller, S. Influence of lipid bilayer properties on nanodisc formation mediated by styrene/maleic acid copolymers. Nanoscale 2016, 8, 15016-15026. [CrossRef] [PubMed]

17. Dominguez Pardo, J.J.; Dorr, J.M.; Iyer, A.; Cox, R.C.; Scheidelaar, S.; Koorengevel, M.C.; Subramaniam, V.; Killian, J.A. Solubilization of lipids and lipid phases by the styrene-maleic acid copolymer. Eur. Biophys. J. 2017, 46, 91-101. [CrossRef] [PubMed]

18. Craig, A.F.; Clark, E.E.; Sahu, I.D.; Zhang, R.; Frantz, N.D.; Al-Abdul-Wahid, M.S.; Dabney-Smith, C.; Konkolewicz, D.; Lorigan, G.A. Tuning the size of styrene-maleic acid copolymer-lipid nanoparticles (SMALPs) using RAFT polymerization for biophysical studies. Biochim. Biophys. Acta 2016, 1858, 2931-2939. [CrossRef] [PubMed]

19. Voskoboynikova, N.; Mosslehy, W.; Colbasevici, A.; Ismagulova, T.T.; Bagrov, D.V.; Akovantseva, A.A.; Timashev, P.S.; Mulkidjanian, A.Y.; Bagratashvili, V.N.; Shaitan, K.V.; et al. Characterization of an archaeal photoreceptor/transducer complex from Natronomonas pharaonis assembled within styrene-maleic acid lipid particles. Rsc Adv. 2017, 7, 51324-51334. [CrossRef]

20. Gulati, S.; Jamshad, M.; Knowles, T.J.; Morrison, K.A.; Downing, R.; Cant, N.; Collins, R.; Koenderink, J.B.; Ford, R.C.; Overduin, M.; et al. Detergent-free purification of ABC (ATP-binding-cassette) transporters. Biochem. J. 2014, 461, 269-278. [CrossRef]

21. Postis, V.; Rawson, S.; Mitchell, J.K.; Lee, S.C.; Parslow, R.A.; Dafforn, T.R.; Baldwin, S.A.; Muench, S.P. The use of SMALPs as a novel membrane protein scaffold for structure study by negative stain electron microscopy. Biochim. Biophys. Acta 2015, 1848, 496-501. [CrossRef]

22. Parmar, M.; Rawson, S.; Scarff, C.A.; Goldman, A.; Dafforn, T.R.; Muench, S.P.; Postis, V.L.G. Using a SMALP platform to determine a sub-nm single particle cryo-EM membrane protein structure. Biochim. Biophys. Acta 2018, 1860, 378-383. [CrossRef] [PubMed]

23. Sun, C.; Gennis, R.B. Single-particle cryo-EM studies of transmembrane proteins in SMA copolymer nanodiscs. Chem. Phys. Lipids 2019, 221, 114-119. [CrossRef] [PubMed]

24. Oluwole, A.O.; Danielczak, B.; Meister, A.; Babalola, J.O.; Vargas, C.; Keller, S. Solubilization of Membrane Proteins into Functional Lipid-Bilayer Nanodiscs Using a Diisobutylene/Maleic Acid Copolymer. Angew. Chem. Int. Ed. 2017, 56, 1919-1924. [CrossRef] [PubMed]

25. Oluwole, A.O.; Klingler, J.; Danielczak, B.; Babalola, J.O.; Vargas, C.; Pabst, G.; Keller, S. Formation of Lipid-Bilayer Nanodiscs by Diisobutylene/Maleic Acid (DIBMA) Copolymer. Langmuir 2017, 33, 14378-14388. [CrossRef] [PubMed]

26. Swainsbury, D.J.K.; Scheidelaar, S.; Van Grondelle, R.; Killian, J.A.; Jones, M.R. Bacterial Reaction Centers Purified with Styrene Maleic Acid Copolymer Retain Native Membrane Functional Properties and Display Enhanced Stability. Angew. Chem. Int. Ed. 2014, 53, 11803-11807. [CrossRef] [PubMed]

27. Barniol-Xicota, M.; Verhelst, S.H.L. Stable and Functional Rhomboid Proteases in Lipid Nanodiscs by Using Diisobutylene/Maleic Acid Copolymers. J. Am. Chem. Soc. 2018, 140, 14557-14561. [CrossRef]

28. Lee, A.G. How lipids affect the activities of integral membrane proteins. Biochim. Biophys. Acta 2004, 1666, 62-87. [CrossRef] [PubMed]

29. Inagaki, S.; Ghirlando, R.; White, J.F.; Gvozdenovic-Jeremic, J.; Northup, J.K.; Grisshammer, R. Modulation of the interaction between neurotensin receptor NTS1 and Gq protein by lipid. J. Mol. Biol. 2012, 417, 95-111. [CrossRef] [PubMed]

30. Soubias, O.; Gawrisch, K. The role of the lipid matrix for structure and function of the GPCR rhodopsin. Biochim. Biophys. Acta 2012, 1818, 234-240. [CrossRef] [PubMed]

31. Rietveld, A.; Berkhout, T.A.; Roenhorst, A.; Marsh, D.; De Kruijff, B. Preferential association of apocytochrome c with negatively charged phospholipids in mixed model membranes. Biochim. Biophys. Acta 1986, 858, 38-46. [CrossRef]

32. Wolfs, J.A.; Horvath, L.I.; Marsh, D.; Watts, A.; Hemminga, M.A. Spin-label ESR of bacteriophage M13 coat protein in mixed lipid bilayers. Characterization of molecular selectivity of charged phospholipids for the bacteriophage M13 coat protein in lipid bilayers. Biochemistry 1989, 28, 9995-10001. [CrossRef] [PubMed]

33. Keller, R.C.; Ten Berge, D.; Nouwen, N.; Snel, M.M.; Tommassen, J.; Marsh, D.; De Kruijff, B. Mode of insertion of the signal sequence of a bacterial precursor protein into phospholipid bilayers as revealed by cysteine-based site-directed spectroscopy. Biochemistry 1996, 35, 3063-3071. [CrossRef] [PubMed] 
34. Sahu, I.D.; McCarrick, R.M.; Troxel, K.R.; Zhang, R.F.; Smith, H.J.; Dunagan, M.M.; Swartz, M.S.; Rajan, P.V.; Kroncke, B.M.; Sanders, C.R.; et al. DEER EPR Measurements for Membrane Protein Structures via Bifunctional Spin Labels and Lipodisq Nanoparticles. Biochemistry 2013, 52, 6627-6632. [CrossRef]

35. Orban-Glass, I.; Voskoboynikova, N.; Busch, K.B.; Klose, D.; Rickert, C.; Mosslehy, W.; Roder, F.; Wilkens, V.; Piehler, J.; Engelhard, M.; et al. Clustering and Dynamics of Phototransducer Signaling Domains Revealed by Site-Directed Spin Labeling Electron Paramagnetic Resonance on SRII/HtrII in Membranes and Nanodiscs. Biochemistry 2015, 54, 349-362. [CrossRef] [PubMed]

36. Sahu, I.D.; Kroncke, B.M.; Zhang, R.; Dunagan, M.M.; Smith, H.J.; Craig, A.; McCarrick, R.M.; Sanders, C.R.; Lorigan, G.A. Structural investigation of the transmembrane domain of KCNE1 in proteoliposomes. Biochemistry 2014, 53, 6392-6401. [CrossRef]

37. Stepien, P.; Polit, A.; Wisniewska-Becker, A. Comparative EPR studies on lipid bilayer properties in nanodiscs and liposomes. Biochim. Biophys. Acta 2015, 1848, 60-66. [CrossRef] [PubMed]

38. Mosslehy, W.; Voskoboynikova, N.; Colbasevici, A.; Ricke, A.; Klose, D.; Klare, J.P.; Mulkidjanian, A.Y.; Steinhoff, H.J. Conformational Dynamics of Sensory Rhodopsin II in Nanolipoprotein and Styrene-Maleic Acid Lipid Particles. Photochem. Photobiol. 2019, 95, 1195-1204. [CrossRef]

39. Bali, A.P.; Sahu, I.D.; Craig, A.F.; Clark, E.E.; Burridge, K.M.; Dolan, M.T.; Dabney-Smith, C.; Konkolewicz, D.; Lorigan, G.A. Structural characterization of styrene-maleic acid copolymer-lipid nanoparticles (SMALPs) using EPR spectroscopy. Chem. Phys. Lipids 2019, 220, 6-13. [CrossRef] [PubMed]

40. Hubbell, W.L.; McConnell, H.M. Molecular motion in spin-labeled phospholipids and membranes. J. Am. Chem. Soc. 1971, 93, 314-326. [CrossRef] [PubMed]

41. Subczynski, W.K.; Raguz, M.; Widomska, J. Studying lipid organization in biological membranes using liposomes and EPR spin labeling. Methods Mol. Biol. 2010, 606, 247-269. [CrossRef] [PubMed]

42. Subczynski, W.K.; Wisniewska, A.; Yin, J.J.; Hyde, J.S.; Kusumi, A. Hydrophobic barriers of lipid bilayer membranes formed by reduction of water penetration by alkyl chain unsaturation and cholesterol. Biochemistry 1994, 33, 7670-7681. [CrossRef] [PubMed]

43. Xue, M.; Cheng, L.; Faustino, I.; Guo, W.; Marrink, S.J. Molecular Mechanism of Lipid Nanodisk Formation by Styrene-Maleic Acid Copolymers. Biophys. J. 2018, 115, 494-502. [CrossRef]

44. Orekhov, P.S.; Bozdaganyan, M.E.; Voskoboynikova, N.; Mulkidjanian, A.Y.; Steinhoff, H.J.; Shaitan, K.V. Styrene/Maleic Acid Copolymers Form SMALPs by Pulling Lipid Patches out of the Lipid Bilayer. Langmuir 2019, 35, 3748-3758. [CrossRef] [PubMed]

45. Colbasevici, A.; Voskoboynikova, N.; Orekhov, P.S.; Bozdaganyan, M.E.; Karlova, M.G.; Sokolova, O.S.; Klare, J.P.; Mulkidjanian, A.Y.; Shaitan, K.V.; Steinhoff, H.J. Lipid dynamics in nanoparticles formed by maleic acid-containing copolymers: EPR spectroscopy and molecular dynamics simulations. Biochim. Biophys. Acta Biomembr. 2020, 1862, 183207. [CrossRef]

46. Bagrov, D.V.; Voskoboynikova, N.; Armeev, G.A.; Mosslehy, W.; Gluhov, G.S.; Ismagulova, T.T.; Mulkidjanian, A.Y.; Kirpichnikov, M.P.; Steinhoff, H.J.; Shaitan, K.V. Characterization of lipodisc nanoparticles containing sensory rhodopsin ii and its cognate transducer from Natronomonas pharaonis. Biophysics 2016, 61, 942-949. [CrossRef]

47. Freed, J.H. Theory of slow tumbling ESR spectra for nitroxides. In Spin Labeling: Theory and Applications; Springer: Berlin/Heidelberg, Germany, 1976; Volume 1, pp. 53-132.

48. Marsh, D.; Watts, A.; Pates, R.D.; Uhl, R.; Knowles, P.F.; Esmann, M. ESR spin-label studies of lipid-protein interactions in membranes. Biophys. J. 1982, 37, 265-274. [CrossRef]

49. Shalaeva, D.N.; Galperin, M.Y.; Mulkidjanian, A.Y. Eukaryotic G protein-coupled receptors as descendants of prokaryotic sodium-translocating rhodopsins. Biol. Direct 2015, 10, 63. [CrossRef] [PubMed]

50. Gordeliy, V.I.; Labahn, J.; Moukhametzianov, R.; Efremov, R.; Granzin, J.; Schlesinger, R.; Buldt, G.; Savopol, T.; Scheidig, A.J.; Klare, J.P.; et al. Molecular basis of transmembrane signalling by sensory rhodopsin II-transducer complex. Nature 2002, 419, 484-487. [CrossRef] [PubMed]

51. Chizhov, I.; Schmies, G.; Seidel, R.; Sydor, J.R.; Luttenberg, B.; Engelhard, M. The photophobic receptor from Natronobacterium pharaonis: Temperature and $\mathrm{pH}$ dependencies of the photocycle of sensory rhodopsin II. Biophys. J. 1998, 75, 999-1009. [CrossRef]

52. Wegener, A.A.; Klare, J.P.; Engelhard, M.; Steinhoff, H.J. Structural insights into the early steps of receptor-transducer signal transfer in archaeal phototaxis. Embo J. 2001, 20, 5312-5319. [CrossRef] [PubMed]

53. Klare, J.P.; Bordignon, E.; Engelhard, M.; Steinhoff, H.J. Sensory rhodopsin II and bacteriorhodopsin: Light activated helix F movement. Photochem. Photobiol. Sci. 2004, 3, 543-547. [CrossRef] [PubMed]

54. Schmies, G.; Luttenberg, B.; Chizhov, I.; Engelhard, M.; Becker, A.; Bamberg, E. Sensory rhodopsin II from the haloalkaliphilic natronobacterium pharaonis: Light-activated proton transfer reactions. Biophys. J. 2000, 78, 967-976. [CrossRef]

55. Wegener, A.A.; Chizhov, I.; Engelhard, M.; Steinhoff, H.J. Time-resolved detection of transient movement of helix F in spin-labelled pharaonis sensory rhodopsin II. J. Mol. Biol. 2000, 301, 881-891. [CrossRef] [PubMed]

56. Forte, T.M.; Nichols, A.V.; Gong, E.L.; Levy, R.I.; Lux, S. Electron microscopic study on reassembly of plasma high density apoprotein with various lipids. Biochim. Biophys. Acta 1971, 248, 381-386. [CrossRef]

57. Brouillette, C.G.; Jones, J.L.; Ng, T.C.; Kercret, H.; Chung, B.H.; Segrest, J.P. Structural studies of apolipoprotein AI/phosphatidylcholine recombinants by high-field proton NMR, nondenaturing gradient gel electrophoresis, and electron microscopy. Biochemistry 1984, 23, 359-367. [CrossRef] [PubMed]

58. Thompson, R.F.; Walker, M.; Siebert, C.A.; Muench, S.P.; Ranson, N.A. An introduction to sample preparation and imaging by cryo-electron microscopy for structural biology. Methods 2016, 100, 3-15. [CrossRef] 
59. Dobro, M.J.; Melanson, L.A.; Jensen, G.J.; McDowall, A.W. Plunge freezing for electron cryomicroscopy. Methods Enzymol. 2010, 481, 63-82. [CrossRef] [PubMed]

60. Markvoort, A.J.; Van Santen, R.A.; Hilbers, P.A. Vesicle shapes from molecular dynamicssimulations. J. Phys. Chem. B 2006, 110, 22780-22785. [CrossRef] [PubMed]

61. Chng, C.-P. Effect of simulation temperature on phospholipid bilayer-vesicle transition studied by coarse-grained molecular dynamics simulations. Soft Matter 2013, 9, 7294-7301. [CrossRef]

62. Shillcock, J.C. Spontaneous vesicle self-assembly: A mesoscopic view of membrane dynamics. Langmuir 2012, 28, 541-547. [CrossRef] [PubMed]

63. Alessandri, R.; Souza, P.C.T.; Thallmair, S.; Melo, M.N.; De Vries, A.H.; Marrink, S.J. Pitfalls of the Martini Model. J. Chem. Theory Comput. 2019, 15, 5448-5460. [CrossRef] [PubMed]

64. Sasaki, J.; Spudich, J.L. The transducer protein HtrII modulates the lifetimes of sensory rhodopsin II photointermediates. Biophys. J. 1998, 75, 2435-2440. [CrossRef]

65. Shibata, M.; Inoue, K.; Ikeda, K.; Konno, M.; Singh, M.; Kataoka, C.; Abe-Yoshizumi, R.; Kandori, H.; Uchihashi, T. Oligomeric states of microbial rhodopsins determined by high-speed atomic force microscopy and circular dichroic spectroscopy. Sci. Rep. 2018, 8, 8262. [CrossRef] [PubMed]

66. Hohenfeld, I.P.; Wegener, A.A.; Engelhard, M. Purification of histidine tagged bacteriorhodopsin, pharaonis halorhodopsin and pharaonis sensory rhodopsin II functionally expressed in Escherichia coli. FEBS Lett. 1999, 442, 198-202. [CrossRef]

67. Mennes, N.; Klare, J.P.; Chizhov, I.; Seidel, R.; Schlesinger, R.; Engelhard, M. Expression of the halobacterial transducer protein HtrII from Natronomonas pharaonis in Escherichia coli. FEBS Lett. 2007, 581, 1487-1494. [CrossRef] [PubMed]

68. Klose, D.; Voskoboynikova, N.; Orban-Glass, I.; Rickert, C.; Engelhard, M.; Klare, J.P.; Steinhoff, H.J. Light-induced switching of HAMP domain conformation and dynamics revealed by time-resolved EPR spectroscopy. FEBS Lett. 2014, 588, 3970-3976. [CrossRef] [PubMed]

69. Abraham, M.J.; Murtola, T.; Schulz, R.; Páll, S.; Smith, J.C.; Hess, B.; Lindahl, E. GROMACS: High performance molecular simulations through multi-level parallelism from laptops to supercomputers. SoftwareX 2015, 1, 19-25. [CrossRef]

70. Wassenaar, T.A.; Ingolfsson, H.I.; Bockmann, R.A.; Tieleman, D.P.; Marrink, S.J. Computational Lipidomics with insane: A Versatile Tool for Generating Custom Membranes for Molecular Simulations. J. Chem. Theory Comput. 2015, 11, 2144-2155. [CrossRef]

71. De Jong, D.H.; Baoukina, S.; Ingolfsson, H.I.; Marrink, S.J. Martini straight: Boosting performance using a shorter cutoff and GPUs. Comput. Phys. Commun. 2016, 199, 1-7. [CrossRef]

72. Michaud-Agrawal, N.; Denning, E.J.; Woolf, T.B.; Beckstein, O. MDAnalysis: A toolkit for the analysis of molecular dynamics simulations. J. Comput. Chem. 2011, 32, 2319-2327. [CrossRef] [PubMed]

73. Kholina, E.G.; Kovalenko, I.B.; Bozdaganyan, M.E.; Strakhovskaya, M.G.; Orekhov, P.S. Cationic Antiseptics Facilitate Pore Formation in Model Bacterial Membranes. J. Phys. Chem. B 2020, 124, 8593-8600. [CrossRef] [PubMed] 